\title{
Effects of galvanic distortions on magnetotelluric data: Interpretation and its correction using deep electrical data
}

\author{
Jimmy Stephen ${ }^{1}, \mathrm{~S}$ G Gokarn ${ }^{2}, \mathrm{C} \mathrm{Manoj}^{1}$ and S B Singh ${ }^{1}$ \\ ${ }^{1}$ National Geophysical Research Institute, \# 66, Hyderabad 500 00\%, India. e-mail: jimmy@ngri.res.in \\ ${ }^{2}$ Indian Institute of Geomagnetism, Colaba, Mumbai 400 005, India.
}

The non-inductive galvanic disturbances due to surficial bodies, lying smaller than high frequency skin depth, cause serious interpretational errors in magnetotelluric data. These frequency independent distortions result in a quasi-static shift between the apparent resistivity curves known as static shift. Two-dimensional modelling studies, for the effects of surficial bodies on magnetotelluric interpretation, show that the transverse electric (TE) mode apparent resistivity curves are hardly affected compared to the transverse magnetic (TM) mode curves, facilitating the correction by using a curve shifting method to match low frequency asymptotes. But in the case of field data the problem is rather complicated because of the random distribution of geometry and conductivity of near surface inhomogeneities. Here we present the use of deep resistivity sounding (DRS) data to constrain MT static shift. Direct current sensitivity studies show that the behaviour of MT static shift can be estimated using DC resistivity measurements close to the MT sounding station to appreciable depths. The distorted data set is corrected using the MT response for DRS model and further subject to joint inversion with DRS data. Joint inversion leads to better estimation of MT parameters compared to the separate inversion of data sets.

\section{Introduction}

The development of MT method after Tikhonov (1950) and Cagniard (1953) made a landmark in the application of EM techniques to explore conductive subsurface structures of the Earth. The two orthogonal complex components of horizontal magnetic field $\left(H_{x}, H_{y}\right)$ and the electric fields $\left(E_{x}, E_{y}\right)$ measured at earth's surface (with $X$ pointing north and $Y$ pointing east) are related through the equation in the tensor form,

$$
\left[\begin{array}{l}
E_{x} \\
E_{y}
\end{array}\right]=\left[\begin{array}{ll}
Z_{x x} & Z_{x y} \\
Z_{y x} & Z_{y y}
\end{array}\right]\left[\begin{array}{l}
H_{x} \\
H_{y}
\end{array}\right]
$$

where $Z_{x x}, Z_{x y}, Z_{y x}$ and $Z_{y y}$ are called impedance tensor elements, each of them being a complex quantity. The impedance tensor is the central parameter around which the magnetotelluric interpretations revolve and has all the sub-structural information, which is obtained from the MT technique.
In the case of a horizontally layered earth, $Z_{x x}=$ $Z_{y y}=0$ and $Z_{x y}=-Z_{y x}$, because $E_{x}$ depends only on the perpendicular component $H_{y}$ and is independent of $H_{x}$. In the case of a two-dimensional earth, $Z_{x x}$ and $Z_{y y}$ are non-zero, except when measured either parallel or perpendicular to the strike of the geological formations and vary with the rotation of the coordinate system. The principal impedance elements can be rotated mathematically either parallel (TE or E-polarisation) or perpendicular (TM or H-polarisation) to the strike so as to reduce $Z_{x x}$ and $Z_{y y}$ to zero.

Based on the estimates of tensor impedance elements, the apparent resistivity and phase at different frequencies may be obtained from the following relations.

$$
\begin{aligned}
& \rho_{x y}(\omega)=0.2 \mathrm{~T}\left|Z_{x y}\right|^{2}, \\
& \phi_{x y}(\omega)=\operatorname{Arg}\left(Z_{x y}\right),
\end{aligned}
$$

Keywords. Magnetotelluric; deep resistivity sounding; galvanic distortion; static shift; joint inversion. 
where $\rho_{x y}$ is the apparent resistivity and $\phi_{x y}$ is the phase in the N-S direction, $\omega$ is the angular frequency given by $\omega=2 \pi f, f$ being frequency and $T$ is the period. Thus the final output of processing of data, in general, provides us with the apparent resistivity and phase curves plotted against frequency. The range of application of MT method can be perceived from studies carried out by Orange (1989), Jones (1992), Gokarn et al (1999) and Almeida et al (2001).

The objective of this paper is to discuss various interpretational errors inherent to MT data due to some unpredictable factors. Like any other electromagnetic or electrical methods, since the deep penetrations are possible only for long period signals, the resolvability of any thin layer at depth is difficult. The present study illustrates the major distortions in MT data set leading to a completely erroneous interpretation and discusses the possible corrective measures at the cost of DC sensitivity of MT telluric signals.

\section{Galvanic distortions on MT data}

The distortion of MT sounding curves arises mainly from the telluric field, which is of galvanic or inductive nature as discussed by Berdichevsky and Dmitriev (1976). These effects are independent of the frequency of the wave and are normally of quasi-static character that causes vertical shifts in MT apparent resistivity curves called "static shift". The static shift does not have any effects on the phase variations. Usually the galvanic effects are found to be dominant in MT data except the strong induction effects obtained near deep elongated depression filled with conductive sediments or near water filled deep fractures (which can be recognized by the variations in polarization modes of MT recording and can be corrected by modelling it during the interpretation). The cause of galvanic distortion is the accumulation of space charge along boundaries of shallow inhomogeneities. The major contribution comes from the surficial bodies, which lie within the skin depth at the highest frequency. This affects the entire sounding curve at all frequencies giving rise to static shift.

Studies by Park et al (1983); Wannamaker et al (1984) and; Park (1985) show the importance of galvanic distortions due to $2 \mathrm{D}$ and $3 \mathrm{D}$ bodies. Different types of correction procedures were developed, such as, transient EM methods (Sternberg et al 1988; Pellerin and Hohmann 1990a and 1990b), laterally homogeneous layer constraints from MT data over a region (Jones 1988), utilization of conductivity distribution charts over the region (Demidova et al 1985), shallow resistivity soundings (Romo et al 1997), etc. Studies carried out by Brasse and Rath (1997) show that the finite telluric spreads located very close will also yield significantly shifted MT responses. The combination of DC and EM measurements as well as the joint inversion of deep resistivity sounding (DRS) data with MT sounding data has been reported by Harinarayana $(1993,1999)$. Later the DC sensitivity distribution for finite telluric layouts was studied by Spitzer (2001) to establish that the galvanic effects are merely a DC problem.

The paper has been divided into two parts, the first part comprising a two-dimensional magnetotelluric modelling study to understand the nature of static shifts from $2 \mathrm{D}$ resistive and conductive bodies and the second part comprising a joint approach of correcting the static shift using deep resistivity sounding data. The modelling study has helped to understand the behaviour of static shift at inhomogeneity boundary on both TE and TM apparent resistivity curves. Further, the static shift in the field data was constrained using deep resistivity sounding data since the DC electrical methods are less sensitive to shallow perturbations than MT. Static corrected data was then utilized for estimating layer parameters. Here the joint inversion of DRS and MT sounding data is carried out to provide the better resolution of parameters.

\section{Modelling study}

We have considered 2D inhomogeneous bodies of larger width to show the MT characteristics over the perturbing bodies and at its boundaries. The starting model for generation of the synthetic data is essentially that of a typical stable continental shield, with a $15 \mathrm{~km}$ thick upper crust having a resistivity of $10,000 \mathrm{Ohm}-\mathrm{m}$, overlying a conductive mid-crustal conductor of $5 \mathrm{~km}$ thickness and $20 \mathrm{Ohm}-\mathrm{m}$ resistivity. The lower crust and lithosphere were assumed to have a resistivity of 700 Ohm-m with $100 \mathrm{~km}$ thickness. The Moho discontinuity is not introduced in this model, since this boundary is essentially an elastic discontinuity. The explanations for the existence of midcrustal conductors are given by Haak et al (1991); Jones (1992); Hyndman et al (1993) and Simpson et al (1997) as well. The highest resistivity of the order of $1000 \mathrm{Ohm}-\mathrm{m}$ are associated with the oldest Precambrian cratons, moderate resistivities with adjacent stable areas and the lowest resistivities of less than $100 \mathrm{Ohm}-\mathrm{m}$ with Phanerozoic mobile belts and those areas tectonically active at present. For the present study, the low resistive Phanerozoic case is considered. A very low resistivity of $10 \mathrm{Ohm}-\mathrm{m}$ at depths of $120 \mathrm{~km}$, resembling the presence of partial melts having liquid phase of 
about 1 to 3\% (Jones 1992), was invoked beneath the lithosphere to represent the conductive boundary separating the lithosphere and asthenosphere. Finally, to resemble a geoelectric model of Deccan volcanic province or the regions of Precambrian sedimentary formations, an $800 \mathrm{~m}$ thick conductive overburden with a resistivity of $100 \mathrm{Ohm}-\mathrm{m}$ was placed on the upper crust.

In the modelling process, the synthetic MT responses, i.e., frequency variation of apparent resistivity and phase, were generated using a finite element two-dimensional forward modelling scheme (Wannamaker et al 1987) over a fixed set of 11 stations (figure 1a). The MT forward response apparent resistivity curves obtained in the absence of any shallow perturbing bodies seem to be undistorted and the TE and TM components are identical and is the same as the one shown in figure 1 for a station far away from the body (station: $-30,000 \mathrm{~m})$.

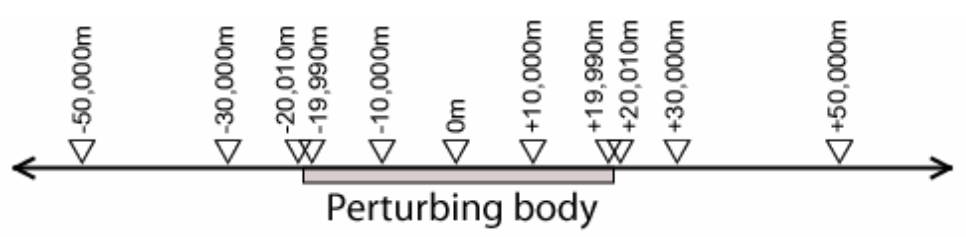

(a)
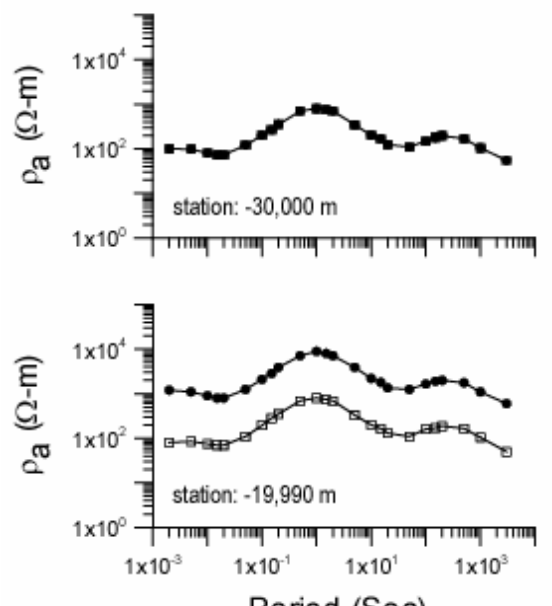

Period (Sec)
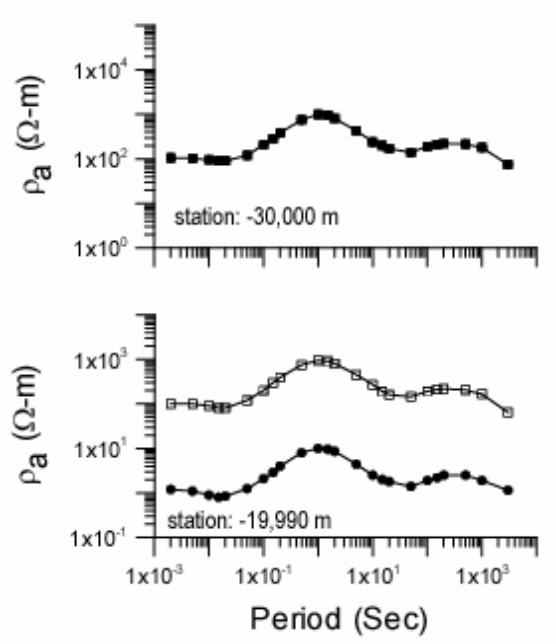
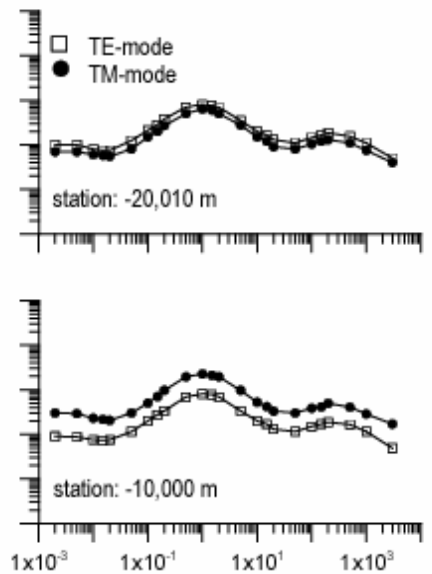

Period (Sec)

(b)
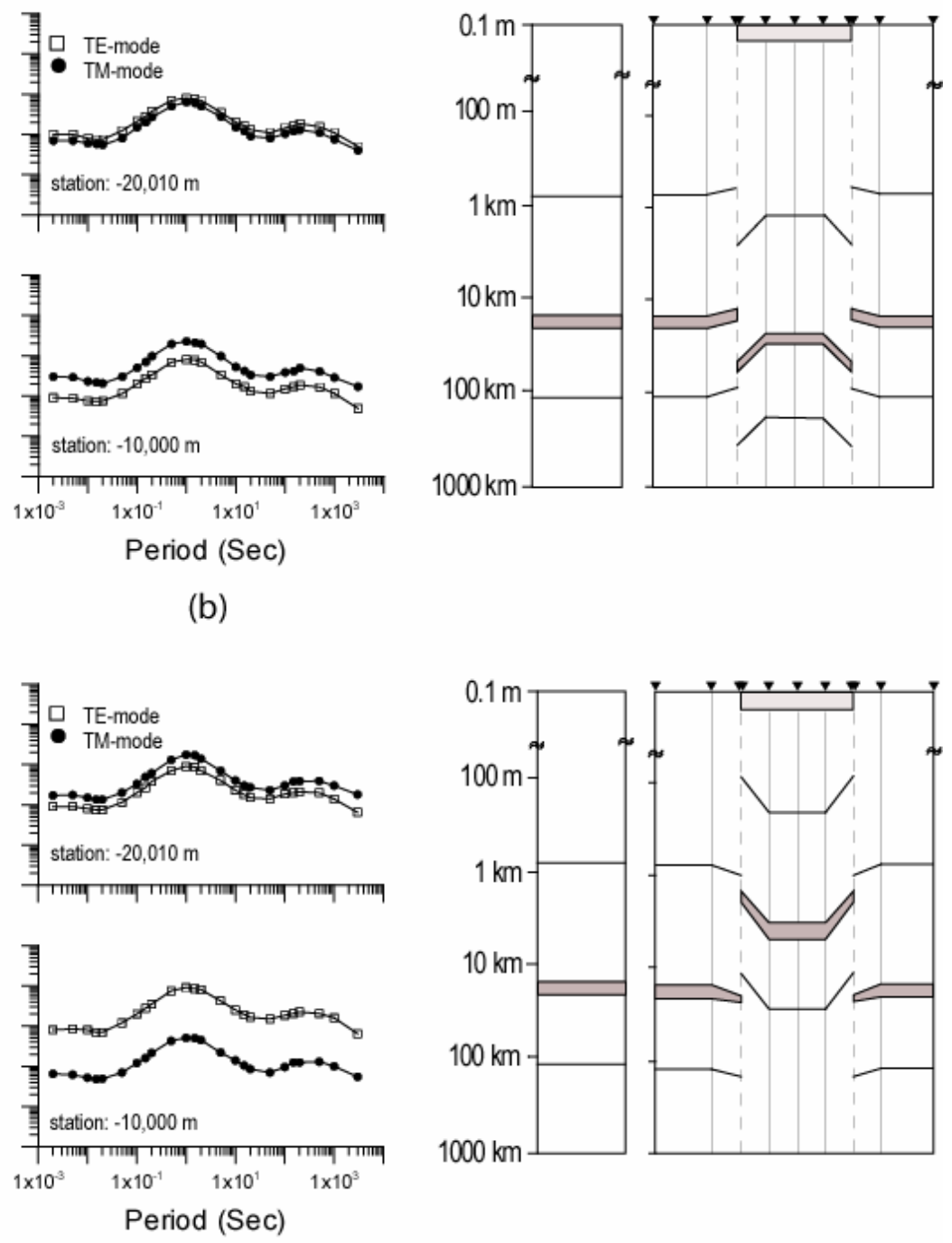

(c)

Figure 1. Modeling results: (a) Layout of stations and relative position of perturbing body used for modelling; (b \& c) Modelling results for resistive and conductive bodies respectively. Both the forward response apparent resistivity curves and the $1 \mathrm{D}$ models (actual and distorted, TM) are also shown. 
Next we introduced the perturbing 2D bodies in the model. Initially, a resistive body of resistivity $1000 \mathrm{Ohm}-\mathrm{m}$ having thickness $20 \mathrm{~m}$ and thereafter, a conductive body of $1 \mathrm{Ohm}-\mathrm{m}$ and $1 \mathrm{~m}$ is invoked. Figure 1 shows the geometry of all the 11 stations with respect to the shallow perturbing body as well as the MT response curves obtained along some of the stations. Here, it is noticed that, in both the resistive and conductive cases, a station out from the inhomogeneous body (station $-30,000 \mathrm{~m}$, at a distance of $10 \mathrm{~km}$ from the body) is not affected. Both the TE and TM responses are the same and reflect the actual model, devoid of any inhomogeneities. But on approaching the perturbing body, the MT curves show distortions in the form of static shift. This shift is different at different locations with respect to the proximity to the boundary. It is noticed in this $2 \mathrm{D}$ modelling that the TE components are devoid of any galvanic charge effects and hence we observe an unaffected apparent resistivity curve. On the other hand, the TM components are highly distorted. Also at stations in the immediate vicinity of body boundaries the shift depends on the negative and positive sensitivity characteristics, giving rise to opposite shifts on either side of the boundary as observed in figure 1(b) and 1(c). The large discrepancies of shifts can visualise at the near boundary stations with a higher shift towards the inward side of the body (as seen for $-19,990 \mathrm{~m}$ ) compared to the outward side $(-20,010 \mathrm{~m})$. Further, the reversing nature of the responses can be seen with the reversing of the body's conductivity property. This is attributed to the DC sensitivity distributions in the resistive and conductive regions, discussed later.

Present modelling studies show that the boundary charge effects are more in the case of conductive body with greater static shifts as seen in figure 1. Since the responses behave differently on both sides of the inhomogeneity boundary, as well as depends on the nature of inhomogeneity, simple conclusions cannot be made on the basis of a static shifted data obtained from field. The nature of inhomogeneity is also unpredictable. The inverted 1D model for resistive and conductive perturbing body models clearly depicts the erroneous estimation of layer parameters in both the cases, resulting in the over-estimation and under-estimation of parameters (figure 1).

The most essential procedure to correct for the galvanic static distortion is to shift the affected curve to the normal level. If the TE mode is not affected, various correction procedures can be implemented. Here, we use a curve shifting technique, where the low frequency asymptotes to MT apparent resistivity curves are matched, since there may not occur much variation in a deeper layer for two adjacent stations. This process may not be so simple, if noises as well as other telluric distortions, such as current channelling affect the MT measurements. Since the bottom of the resistive layer is well constrained in the electromagnetic methods, an asymptote drawn along the decreasing trend in apparent resistivity curve is preferable. Figure 2 ( $a$ and $b$ ) shows the application of the asymptote method to correct static distortions for resistive and conductive perturbing bodies respectively. The asymptote is drawn on the distorted TE or TM apparent resistivity curve, for the prominent resistive layer, appearing steady for both the components. The undistorted phase for both polarizations is shown in the figure as well.

\section{DC approach to the problem}

To deal with real MT data, the reliability of integrating deep electrical data has been tested in this paper. DC electrical data collected for a maximum Schlumberger current electrode spread of $10 \mathrm{~km}$ has been used for correcting MT data, since DC data assumes good control with more resolvability in the shallow part. But, how does shallow conductivity heterogeneity affects the DC data? Recent studies carried out by Spitzer (2001) give a detailed picture of DC analogue to the MT static shift. The DC sensitivity distribution studies show that in the case of Schlumberger sounding, there exists a region of positive and negative sensitivity, which approximates a typical MT set-up at larger current electrode separation. Any inhomogeneous body in the sensitivity region will affect the potential measurements considerably, giving rise to a constant vertical shift. The quantitative expression relating DC and MT static shift factors, linear DC and quadratic MT variations, with the perturbation voltages, is given as,

$$
f^{\mathrm{DC}}=\sqrt{f^{\mathrm{MT}}} .
$$

This relation implies that the magnitude of galvanic effects on MT and DC apparent resistivity curves due to the same perturbing bodies, for the constant telluric field electrodes are different (MT more and DC less). Therefore, DC measurements will give the shallow resistivity distribution more confidently. This gives an opportunity to correct the MT data.

\section{Correcting the field MT data}

Here we present the combined interpretation of MT (Harinarayana et al 2002) and DRS data sets measured over the Southern Granulite Terrain (SGT) 


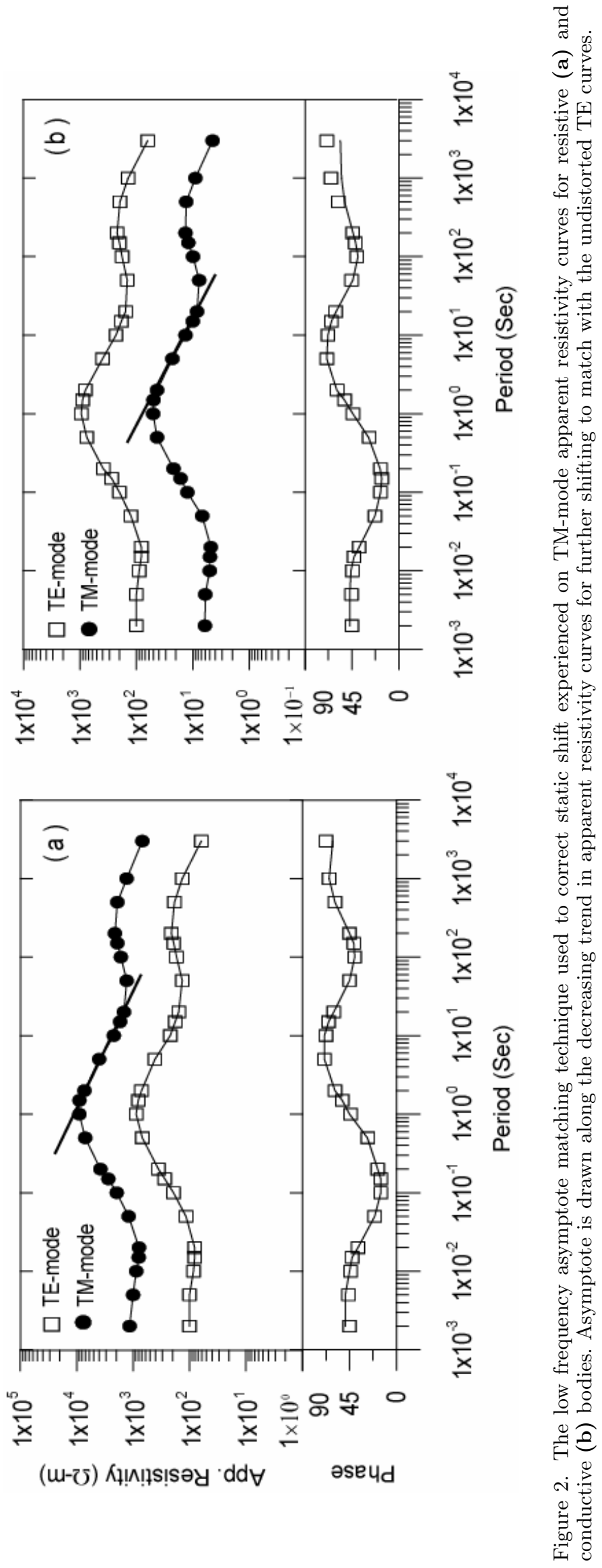



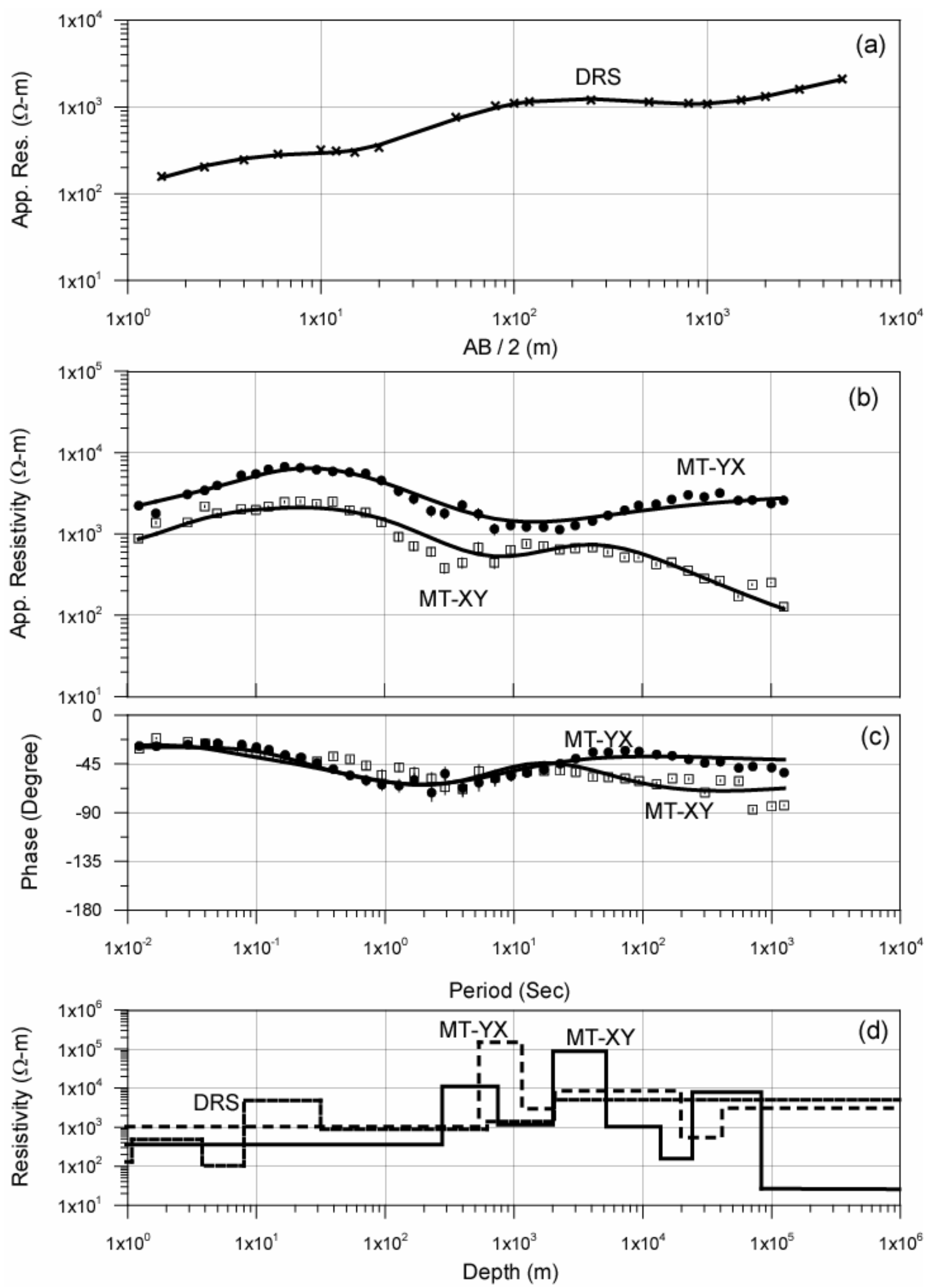

Figure 3. One-dimensional modelling results of DRS and MTS data sets. Observed and computed apparent resistivity curves for DRS (a) and for MT orthogonal components (b) are shown. The phase curves (c) and 1D models obtained on independent interpretation (d) are also given. 


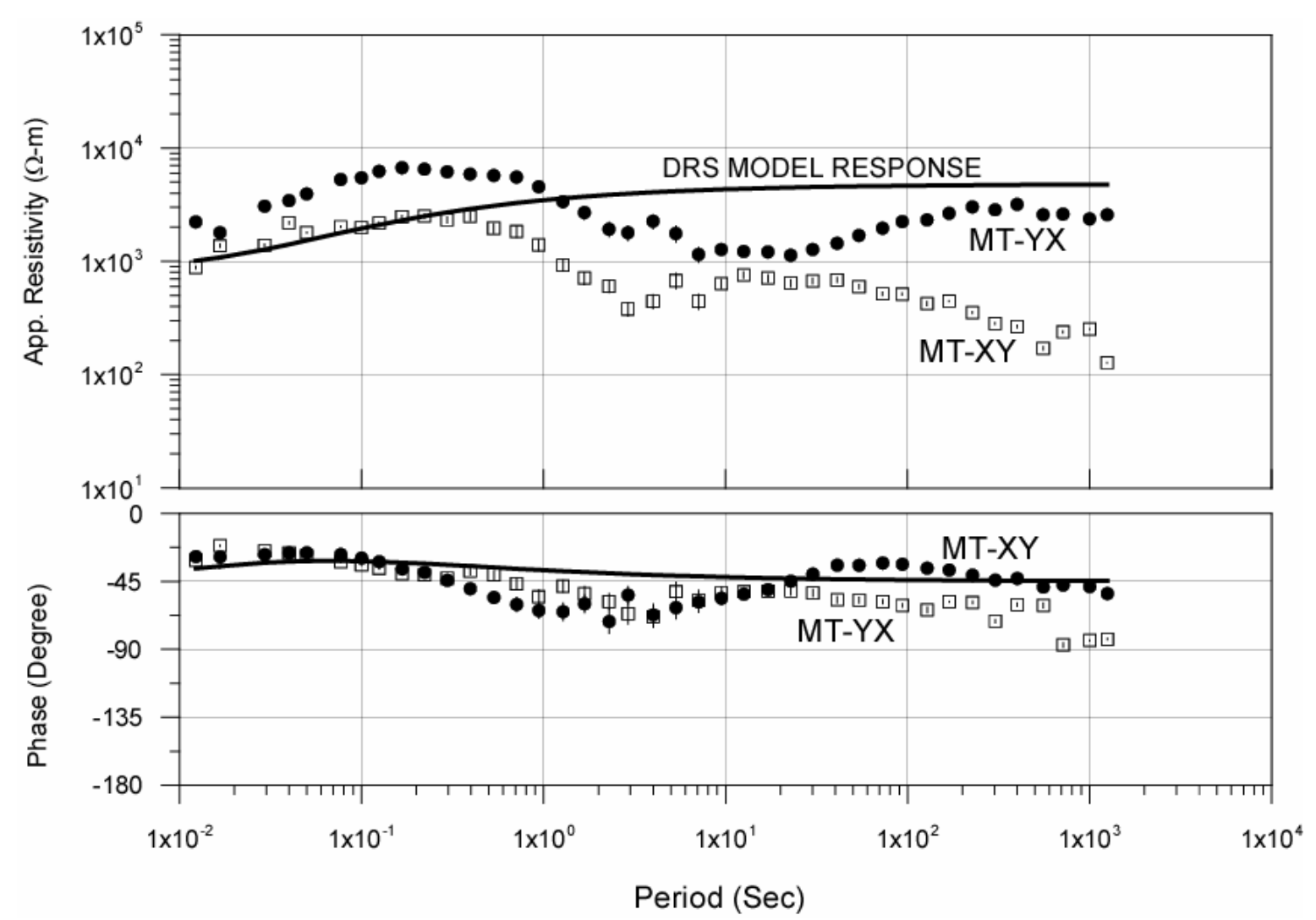

Figure 4. MT response obtained for the DRS layer model to analyse the static shift effects on MTS curves. At higher frequency the DRS model response is well coinciding with the MT- $X Y$ apparent resistivity curve indicating this particular curve is not affected, whereas the MT- $Y X$ component experiences a shift. Here $X Y$ component does not need any correction while the $Y X$ component should be corrected.

of India. The DRS data were collected at a station close to the MT site using the Schlumberger set-up with a maximum spread of $10 \mathrm{~km}$. Figure 3 shows both the DRS and MT $\left(\rho_{x y}\right.$ and $\left.\rho_{y x}\right)$ data set with independent one-dimensional models. In this particular example both the galvanic and induction effects are clearly seen in the MT apparent resistivity curves. The vertical DC shift observed throughout the curves between $X Y$ and $Y X$ components show the galvanic distortion, whereas the diverging nature observed at longer periods depict the deeper resistivity anisotropy. This is also supported by the phase data, which shows split only at longer periods. The model derived by DRS data shows some shallow resistivity perturbations. The $1 \mathrm{D}$ sections shown in figure 3 reflect the level of error in independent interpretation. Both the sections obtained from TE and TM components show considerable dissimilarity even in the shorter period.

We use the DRS data to constrain over the MT data. The MT response for DRS 1D model is presented along with both the components of MT apparent resistivity data (figure 4). In the absence of any static shift, all the responses should match at the higher frequency side. In the present case one could observe a reasonably good match between the MT response of DRS model and the $X Y$ apparent resistivity curve, giving evidence that the $X Y$ component is not being significantly affected by galvanic distortions.

The same asymptote shifting method used for the synthetic data works in the present situation so that an asymptote to the bottom of a prominent high resistive layer is drawn on the affected $Y X$ apparent resistivity curve (figure 5a) and is further shifted to the unaffected curve. The shifted curve with phase (figure 5b and c) along with independent 1D models for DRS, MT- $X Y$ and shifted MT- $Y X$ (figure $5 \mathrm{~d}$ ) are shown. The dissimilarity of $1 \mathrm{D}$ models between $X Y$ and $Y X$ components are very clear even after the static shift correction. Therefore, the final interpretations were done on the basis of the best apparent resistivity curve that keeps maximum consistency with the phase. On observing the apparent resistivity and phase curves (figure $5 \mathrm{~b}$ and $\mathrm{c}$ ), the static corrected $Y X$ curves appear to be more reliable at all frequencies and hence chosen for further interpretation. Joint inversion of DRS and the $Y X$ component of MT apparent resistivity data are carried out using the algorithms given by Jupp and Vozoff 

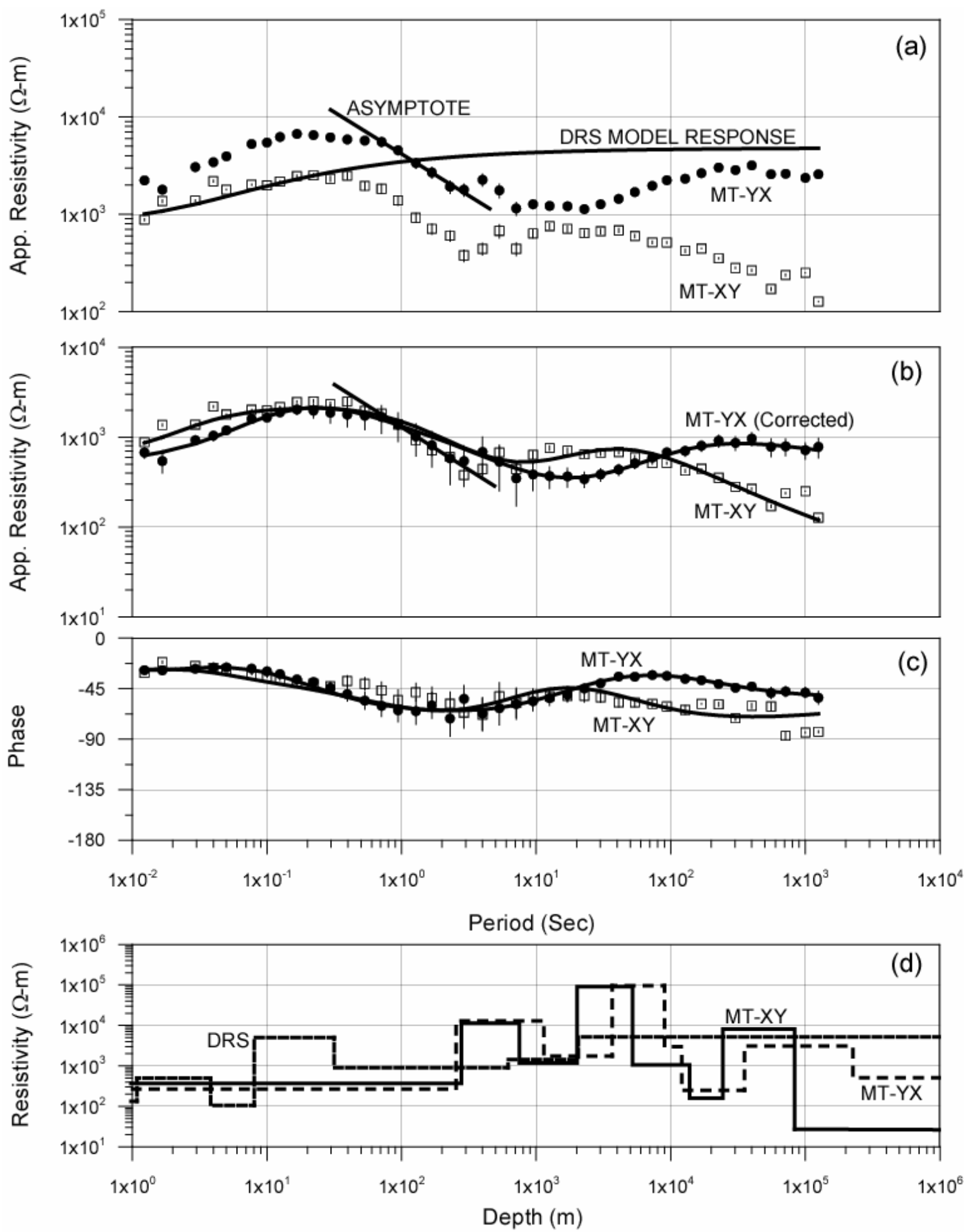

Figure 5. The low frequency asymptote matching procedure. An asymptote along the decreasing trend of the resistivity curve is drawn on the distorted curve (a). This curve is then shifted to the undistorted curve so that the same asymptote coincides with this curve (b). The phase relations (c) as well as the independent 1D models after static correction (d) are shown.

(1975). Here both the DRS and MT sounding apparent resistivity data are inverted simultaneously (applying constraints on each other) and the model is refined to match both the model responses with the observed data sets. Apparent resistivity and phase responses obtained through the joint inversion technique for corrected MT- $Y X$ data, DRS data and the final interpreted geoelectric model are shown in figure 6 . The obtained model exhibits more resolution both at the high frequency 

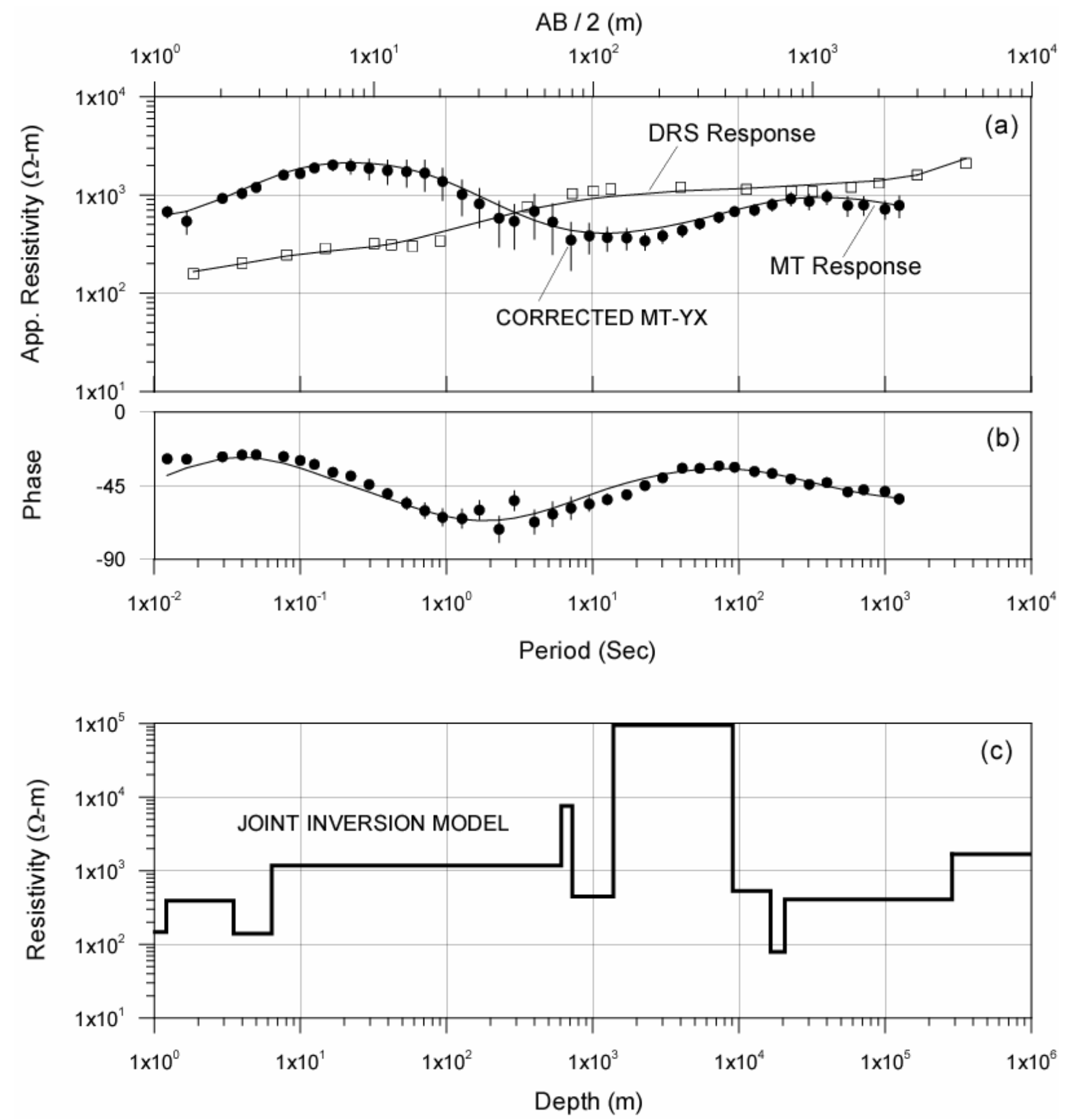

Figure 6. Joint inversion results for DRS and MTS data. The minimum distorted $Y X$ component is taken for joint inversion. The static corrected MT data and computed data for MT and DRS data with observed data (a) and the phase (b) are shown. The result shows very good resolution of layer parameters (c) at shorter and longer periods.

and long period signals than separately inverted models.

\section{Conclusions}

We illustrated various distortions normally seen in magnetotelluric sounding data due to galvanic and inductive current effects. The galvanic effect which is dominant in MT sounding apparent resistivity curves are studied using 2-dimensional modelling over a 1D layered earth on introducing perturbing conductive and resistive bodies. The behaviour of static shift studied along a number of stations with respect to the perturbing inhomogeneity shows different magnitudes of shift, both giving erroneous estimation of layer parameters according to the position of body relative to negative and positive sensitivity region. In the case of $2 \mathrm{D}$ bodies, the TE-mode apparent resistivity curves are free from distortions and the phase is not affected in both TE-mode and TM-mode curves. 
The low frequency asymptote curve shifting method has been implemented to correct the distorted curve, making use of the unaffected curve normally obtained only in the case of a $2 \mathrm{D}$ perturbing body. Though the behaviour of MT sounding curves shown in the present field example taken from the southern granulite terrain of India approximates with the nature of response curves modelled for $2 \mathrm{D}$ perturbing bodies, a more complicated case should be expected always since many shallow bodies represent a 3D geometry rather than a 2D geometry. The asymptote approach using DRS and MT data set are found to be effective in estimating the effects of static shift on both $X Y$ and $Y X$ apparent resistivity curves. Further, the joint inversion of corrected MT data set with DRS data gives more reliable 1D geoelectric section of the subsurface layers with a higher degree of confidence that is not obtained with independent $1 \mathrm{D}$ inversion schemes.

\section{Acknowledgements}

The authors thank the Director, NGRI, for granting permission to publish this work. The first author, Mr. Jimmy Stephen, greatly acknowledges the CSIR for its support, in the form of a Senior Research Fellowship.

\section{References}

Almeida E, Jaume Pous, Santos F M, Fonseca P, Marcuello A, Queralt P, Nolasco R and Victor L M. 2001 Electromagnetic imaging of a transpressional tectonics in SW Iberia; Geophys. Res. Letters 28 439-442

Berdichevsky M N and Dmitriev V I 1976 Distortion of magnetic and electric fields by near surface lateral inhomogeneities; Acta Grodaet. Geophys. Et Mantanist. Acad. Sci. Hung. 11 217-221

Brasse H and Rath V 1997 Audiomagnetotelluric investigations of shallow sedimentary basins in northern Sudan; Geophys. J. Int. 128 301-314

Cagniard L 1953 Basic theory of the magnetotelluric method of geophysical prospecting; Geophysics 18 605-635

Demidova T A, Yegorov I V and Yanikyan V O 1985 Galvanic distortions of the magnetotelluric field of the lower Caucasus; Geomagnetic Aeron. 25 391-396

Gokarn S G, Rao C K, Gautham Gupta and Singh B P 1999 Magnetotelluric techniques; In: Deep Continental Studies in India, DST Newsletter 9(1) 2-8

Haak V, Stoll J and Winter H 1991 Why is the electrical resistivity around the KTB hole so low?; Phys. Earth Planet. Int. 66 12-33
Harinarayana T 1993 Application of joint inversion technique to deep resistivity and magnetotelluric sounding data in Northumberland Basin, Northern England; Geol. Soc. India Mem. 24 113-120

Harinarayana T 1999 Combination of EM and DC measurements for upper crustal studies; Surv. Geophys. 20 257278

Harinarayana T, Naganjaneyulu K, Manoj C, Patro B P K, Begum S K, Murthy D N, Rao M, Kumaraswamy V T C and Virupakshi G. 2002 Magnetotelluric investigations along Kuppam-Palani Geotransect, south India - 2D modeling results; Mem. Geol. Soc. India (in press)

Hyndman R D et al 1993 The origin of electrically conductive lower continental crust: Saline water or graphite?; Phys. Earth Planet. Int. 81 325-344

Jones A G 1988 Static shift of magnetotelluric data and its removal in a sedimentary basin environment; Geophysics 53 967-978

Jones A G 1992 Electrical conductivity of the continental lower crust; In: Continental lower crust (ed) D M Fountain, R J Arculus and R W Kay (Amsterdam: Elsevier) 81-143

Jupp D L B and Vozoff K 1975 Stable iterative methods for the inversion of geophysical data; Geophys. J. R. Astr. Soc. 42 957-976

Orange A S 1989 Magnetotelluric exploration for Hydrocarbons; Proc. of the IEEE 77 287-317

Park S K, Orange A S and Madden T R 1983 Effects of three dimensional structure on magnetotelluric sounding curves; Geophysics 48 1402-1405

Park S K 1985 Distortion of magnetotelluric sounding curves by three dimensional structures; Geophysics $\mathbf{5 0}$ 786-797

Pellerin L and Hohmann G W 1990a Transient electromagnetic inversion: A remedy for magnetotelluric static shifts; Geophysics 55 1242-1250

Pellerin L and Hohmann G 1990b Remedy for static shift; Geophysics 56 951-960

Romo J M, Flores C, Vega R, Vazquez R, Perez Flores M A, Gomez-Trevino E, Esparza F Z, Quizano J E and Garcia V H 1997 A closely spaced magnetotelluric study of the Ahuachapan-Chipilapa geothermal fields, El Salvador; Geothermics $\mathbf{2 6} 627-656$

Simpson F L, Haak V, Khan M A, Sakkas V and Meju M 1997 The KRISP-94 magnetotelluric survey of early 1995: first results; Tectonophysics $\mathbf{2 7 8} 261-271$

Spitzer K 2001 Magnetotelluric static shift and direct current sensitivity; Geophys. J. Int. 144 289-299

Sternberg B K, Washburne J C and Pellerin L 1988 Correction for the static shift in magnetotellurics using transient electromagnetic soundings; Geophysics $\mathbf{5 3}$ $1459-1468$

Tikhonov A 1950 On determining electrical characteristics of the deep layers of the earth's crust; Dokl. Akad. Nauk SSSR 73 295-297

Wannamaker P E, Hohmann G W and Ward S H 1984 Magnetotelluric responses of three-dimensional bodies in layered earths; Geophysics 49 1517-1533

Wannamaker P E, Stodt J A and Rijo L A 1987 Stable finite element solution for two-dimensional magnetotelluric modelling; Geophys. J. Astr. Soc. 88 277296 